\title{
Evaluating the Content and Quality of Emergency Medical Services Oral Patient Handoff Reports
}

\author{
Matthew D. Monaco, Jordan J. Grove, Joshua Beckedorf, Walter A. Schrading
}

Keywords: emergency department, handoff, communication, collegiate-based emergency medical services

Citation (AMA Style): Monaco MD, Grove JJ, Beckedorf J, Schrading WA. Evaluating the Content and Quality of Emergency Medical Services Oral Patient Handoff Reports. J Coll Emerg Med Serv. 2021; 4(2): 16-21. https://doi.org/10.30542/JCEMS.2021.04.02.03

Electronic Link: https://doi.org/10.30542/JCEMS.2021.04.02.03

Published Online: October 31, 2021

Published in Print: October 31, 2021 (Volume 4: Issue 2)

Copyright: @ 2021 Monaco, Grove, Beckedorf \& Schrading. This is an OPEN ACCESS article distributed under the terms of the Creative Commons Attribution 4.0 International (CC BY 4.0) License, which permits unrestricted use, distribution, and reproduction in any medium, provided the original author and source are credited. The full license is available at: www. creativecommons.org/licenses/by/4.0/ 


\title{
Evaluating the Content and Quality of Emergency Medical Services Oral Patient Handoff Reports
}

\author{
Matthew D. Monaco, MD; Jordan J. Grove, MD; Joshua Beckedorf, MD; Walter A. Schrading, MD, FACEP, \\ FAWM
}

\begin{abstract}
Background: Annually millions of patients are brought to Emergency Departments (ED) across the US by air and ground ambulance. There exists no universal criterion for the patient data to be presented from Emergency Medical Services (EMS) to ED personnel. Prior studies show gaps in patient data during handoffs. Objectives: This study seeks to ascertain what patient data is orally reported by EMS to ED personnel. Methods: This was a prospective observational study using a convenience sample of EMS to ED transfer events documented by trained observers. Oral handoffs were evaluated for the presence of selected patient data metrics, interruptions, and whether additional data was obtained through follow-up questions. Results: In total, 102 handoffs from EMS to ED personnel were observed; 76 handoffs were medical and 26 handoffs were traumas. Chief complaints and history of present illness (HPI) were reported most frequently (94\% and 84\% respectively). Vital signs were presented initially during $44 \%$ of cases. Following the initial patient presentation, vital signs were the most requested data on follow-up questioning by receiving medical personnel (15\% of cases). Trauma patients had their physical exam, assessment, and interventions reported more frequently than medical patients but vital signs were reported slightly less so. Conclusions: The frequent absence of patient data metrics like vital signs represents possible limitations in the comprehensiveness of handoffs received by ED personnel from EMS. There appear to be opportunities to improve communication of certain key physical exam findings like vital signs.
\end{abstract}

Keywords: emergency department, handoff, communication, collegiatebased emergency medical services

Corresponding Author and Author Affiliations: Listed at the end of this article.
$\mathrm{I}$ $\mathrm{n}$ the United States in 2016, 22 million patients representing $15.8 \%$ of all Emergency Department (ED) patients arrived via ambulance. ${ }^{1}$ This proportion was greater for patients 75 years and older for whom $40.7 \%$ arrived at the ED via ambulance. ${ }^{1}$ Communication between prehospital providers (EMTs and paramedics) to ED staff is crucial in improving patient safety and reducing medicolegal risk. ${ }^{2}$

Walter Schrading, MD, FACEP, FAWM is an Associate Professor of Emergency Medicine at the University of Alabama at Birmingham. He has been teaching emergency medicine for over 30 years. He is the author of over 30 publications. As the Director of the Office of Wilderness Medicine, he teaches at numerous wilderness medicine courses and runs a specialty track in wild med for EM residents. He has been actively involved in mentoring numerous medical student research projects in emergency medicine. Joshua Beckedorf, MD is a graduating third year resident and upcoming clinical instructor of Emergency Medicine at the University of Alabama at Birmingham. His interests include Wilderness Medicine and he helps teach wilderness medicine courses to medical students and other healthcare personnel. Matthew D Monaco, MD is a prior EMT-B who volunteered providing medical aid and serving as the president of his college's all student run Rensselaer Polytechnic Institute Ambulance. He served for two years after college in the United States Navy as a Surface Warfare Officer. He has recently graduated the University of Alabama School of Medicine and is an incoming emergency medicine intern physician at Naval Medical Center Portsmouth in Portsmouth, VA. Jordan J Grove, MD has recently graduated the University of Alabama School of Medicine and is an incoming emergency medicine intern physician at San Antonio Military Medical Center. Prior to medical school he served as an officer and instructor pilot in the USAF since 2010.
The American College of Emergency Physicians and The National Highway Traffic Safety Administration (NHTSA) provides recommendations for information to be discussed during patient handoffs which include but are not limited to the chief complaint, vital signs, physical exam, and interventions performed by EMS. ${ }^{2,3}$

The success rate of these recommendations was examined in a previous pilot study in which 105 EMS personnel were surveyed on the EMS to ED hand-off with only $42 \%$ having reported receiving standardized training on the hand-off with $35 \%$ concluding that their typical hand-offs are complete. ${ }^{4}$ The above-referenced source illustrates a lack of standardization across disciplines that lead to a disparity of data reported.

Several studies have consistently shown the incompleteness of EMS to ED hand-offs. One study of critically ill patient handoffs from prehospital providers to ED staff found that less than $60 \%$ of handoffs included a complete set of patient vitals. ${ }^{5}$ Another study found that complete vital signs were given in only $53 \%$ of pediatric resuscitation cases in an emergency room and that these handoffs were further complicated by frequent interruptions and redundant questioning between EMS and ED personnel. ${ }^{6}$ In another study that observed over 600 medical hand-offs and 400 trauma hand- 
offs, it was found that the most frequently transferred data during hand-offs was events (99\% trauma, 98.1\% medical) and problems (99\% trauma, 97.5\% medical) while less frequently reported were patient allergies (around 70\%) and vital signs (70-85\% of cases). ${ }^{7}$

Previous studies have focused only on critically ill patients., ${ }^{5,6}$ One of the few studies to date that has included a large number of handoffs and medical and trauma patients was conducted over 10 years ago, between 2010-2011. ${ }^{7}$ With a limited number of studies conducted in this area, especially ones considering non-critically ill patients and involving a vital safety aspect of patient care, this area deserves further study. We hypothesized that certain patient metrics are not being transferred in a substantial number of cases.

\section{Methods}

\section{Study design}

We performed a prospective observational study of handoffs from prehospital providers to ED staff using a convenience sample.

\section{Study setting}

Research was conducted on the campus of a large city-based university emergency department with a level I trauma center which sees over 180,000 patients per year. Data were collected in each of the ED's primary receiving pods 2, 3, 4, and 5 (Trauma Bay). Data was collected from late April through June 2019. A total of 102 handoffs were observed during this time. This was a convenience sample that occurred when trained observers were available and in the department from 0900-2000.

\section{Participants/patients}

This study was reviewed by the Institutional Review Board and applied for and received a designation as Not Human Subjects Research. Patient handoffs were included in the study if patients were at least 18 years of age, not pregnant, and not incarcerated. EMS crews observed were both public and private ambulance services operating in the local area. Both air and ground EMS crews were observed. As above, patients were selected consecutively as part of a convenience sample

\section{Data collection}

Data collection was performed by an experienced emergency medicine resident and two medical students. Medical students were trained by a board-certified emergency physician. Training consisted of covering patient flow in the ED, data to be collected on the data collection sheets, and trial collections in the ED. Students were observed collecting data for accuracy several times before being allowed to independently collect. This was done by having the student and preceptor collect data together on the same case and then comparing results for accuracy.

Data were recorded on standardized data collection forms on which observers circled yes or no as to whether specific data metrics were discussed during patient handoffs from EMS to ED personnel. In this study, a handoff constituted the ongoing and dynamic transfer of patient care as EMS arrives in the ED up until the time they physically leave the patient.

During this study, the observers would await the patient's arrival

Figure 1: Most common paths a patient will take as they are brought in by ambulance to the UED

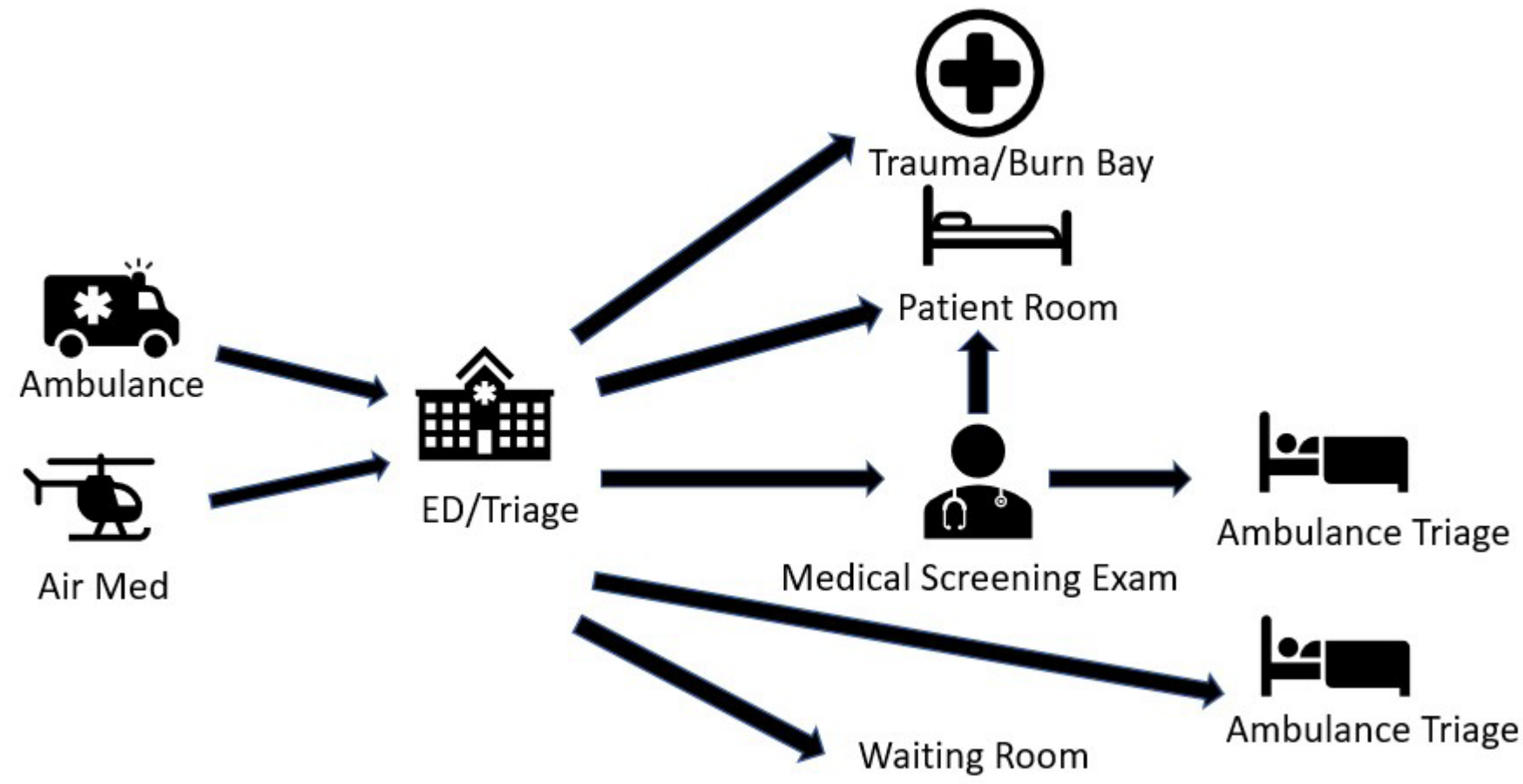

JCEMS · Volume 4 · Issue $2 \cdot$ October 2021 
via ambulance or helicopter. Once the patient had arrived the trained personnel would observe and record the handoffs. A single handoff included all oral reports EMS made on behalf of that individual patient to ED personnel including triage nurses, ED residents/attendings, trauma residents/attendings, and ED nurses. Data transferred during radio transmissions were not included.

Data metrics measured were age, gender, chief complaint, history of present illness (HPI), past/current medical and surgical history, allergies, medications, vital signs, physical exam, EMS Assessment, and EMS interventions performed and or EMS's plan. No identifying patient information was recorded at any time.

Additionally, observers recorded if at any time during a patient handoff 1) the report was interrupted, 2) ED personnel asked for data that was already presented, and 3) for each criterion that was not initially presented if the ED personnel asked for it and if EMS knew the answer.

\section{Results}

A total of 102 patient handoffs between prehospital providers and ED Staff were observed and recorded. 26 were recorded in the trauma and burn bays. Six handoffs were made by Basic Life Support (BLS) crews, 85 by Advanced Life Support (ALS) crews, and 11 by air medical crews.

Figure 2 summarizes the percentage of encounters during which a certain data point was transferred or not. Age and gender were transferred in $19 \%$ and $40 \%$ of cases respectively. Chief complaint and HPI were transferred most often at $94 \%$ and $84 \%$ percent of the time. Historical data metrics including past medical and surgical history (42\%), medications (9\%), and allergies (7\%) were all transferred less often than present illness data and objective and interpretive data.

Objective data including vital signs and physical exams were reported $44 \%$ and $56 \%$ percent of the time. EMS crews provided an assessment of the patient in $44 \%$ of cases. Plan and interventions were likewise reported in $44 \%$ of cases. EMS crews were recorded as having been interrupted during their handoffs $20 \%$ of the time. ED personnel asked EMS crews to repeat information they had already verbally reported $9 \%$ of the time.

As shown in Table 1, following EMS's initial oral reports to ED personnel the most frequently asked questions on follow-up were about vital signs and past medical or surgical history. In 15 of the cases observed EMS crews were asked for the patient's vital signs. Of those 15 cases, EMS crews were able to provide an answer $100 \%$ of the time.

Figure 3 shows a comparison of trauma $(n=26)$ vs medical $(n=76)$ EMS oral reports. The largest discrepancies exist in the rate at which age and gender were reported by EMS crews. Medical patients had their age and gender orally reported $9 \%$ and $26 \%$ of the time while trauma patients had their age and gender reported $46 \%$ and $81 \%$ of the time. Another large discrepancy exists in the rate of past medical and surgical histories provided for patients as EMS crews reported this for medical patients $51 \%$ of the time and for trauma patients $15 \%$ of the time. Physical exam and interventions performed showed crews with trauma patients more often reporting those categories in their oral reports.

\section{Discussion}

The handoff of patients from EMS to ED personnel often occurs

Figure 2: Percentage of time data metrics were transferred by EMS crews during oral handoff reports to the ED

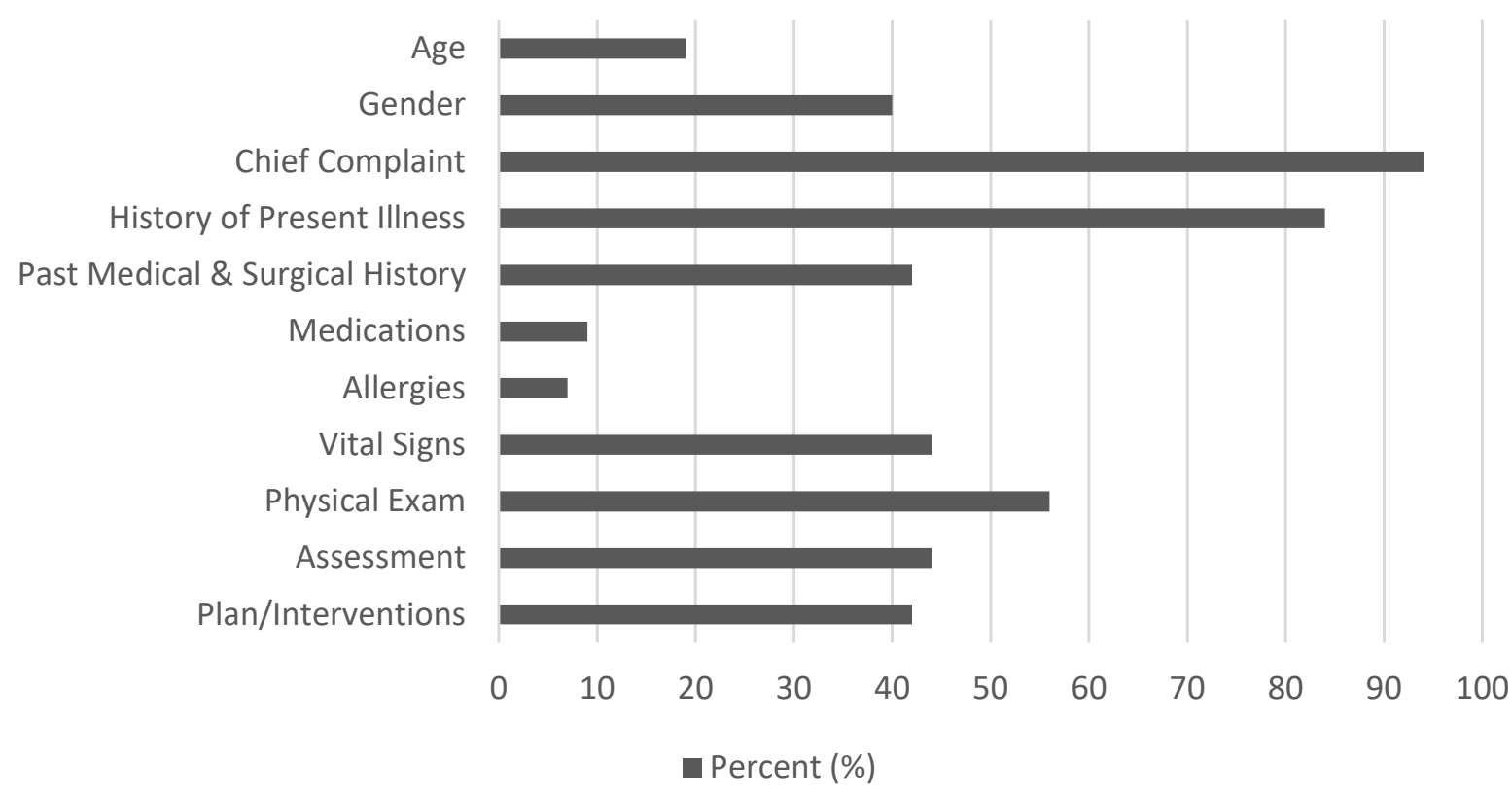


Table 1: Number of times EMS was asked a question about a data point that was not transferred and the number of times they knew the answer

\section{Times Asked Answered/(\%)}

\begin{tabular}{lcc}
\hline Age & 1 & $1(100 \%)$ \\
Gender & 0 & $0(\mathrm{NA})$ \\
Chief Complaint & 1 & $1(100 \%)$ \\
History of Present Illness & 5 & $5(100 \%)$ \\
Past Medical \& Surgical & 13 & $13(100 \%)$ \\
History & 8 & $6(75 \%)$ \\
Medications & 5 & $5(100 \%)$ \\
Allergies & 15 & $15(100 \%)$ \\
Vital Signs & 9 & $9(100 \%)$ \\
Physical Exam & 6 & $6(100 \%)$ \\
Assessment & 9 & $9(100 \%)$ \\
\hline Plan/Interventions &
\end{tabular}

in a dynamic and ever-changing environment. While patient data is often transferred electronically or in written format, oral reports represent the human-to-human transfer of a patient's care from provider to provider. The Joint Commission reported that in 2014 80\% of serious medical errors involved miscommunication between providers during patient transfers of care/handoffs leading to delays in treatment, inappropriate treatments, and increased length of stay in hospitals (8). Oral reports also allow EMS crews to highlight and accentuate areas of concern during their independent role as providers to patients in the prehospital environment and during handoffs.

Our study showed several key results. The first major result being that data metrics such as chief complaint and history of present illness was most often transferred. Objective data such as vital signs and physical exams were less often transferred. The second major issue our study showed is that when considering the cases where EMS did not report vital signs initially, they were prompted for vital signs $26 \%$ of the time by ED staff. In each of the cases where they were asked for vital signs, they were able to provide an answer. Another key result was the comparison of medical vs trauma cases which showed a large discrepancy in the transmission of demographics, physical exam, and assessment which greatly favored EMS crews doing this during traumas. Interestingly, vital signs were verbally reported slightly less often during trauma handoffs. One possible explanation for this is that vital signs were communicated via radio report en route to the hospital for traumas or that most trauma patients are hooked up to monitoring equipment upon arrival to the $\mathrm{ED}$ and during most trauma handoffs. Finally, interruptions to transfers and repeated questions constituted a sizeable number of the handoffs occurring $20 \%$ and $9 \%$ of the time respectively.

Our study concurred with similar studies in that chief complaint was the most often transferred data point. ${ }^{5,6}$ The current literature illustrates vital signs were omitted $50-70 \%$ of the time and past medical history was omitted $40-60 \%$ of the time. ${ }^{5,6}$ Our study showed similar results in regard to omission of vital signs and past medical history. In addition, similar findings

Figure 3: Comparison of the percentage of time data metrics were transferred in trauma vs medical cases

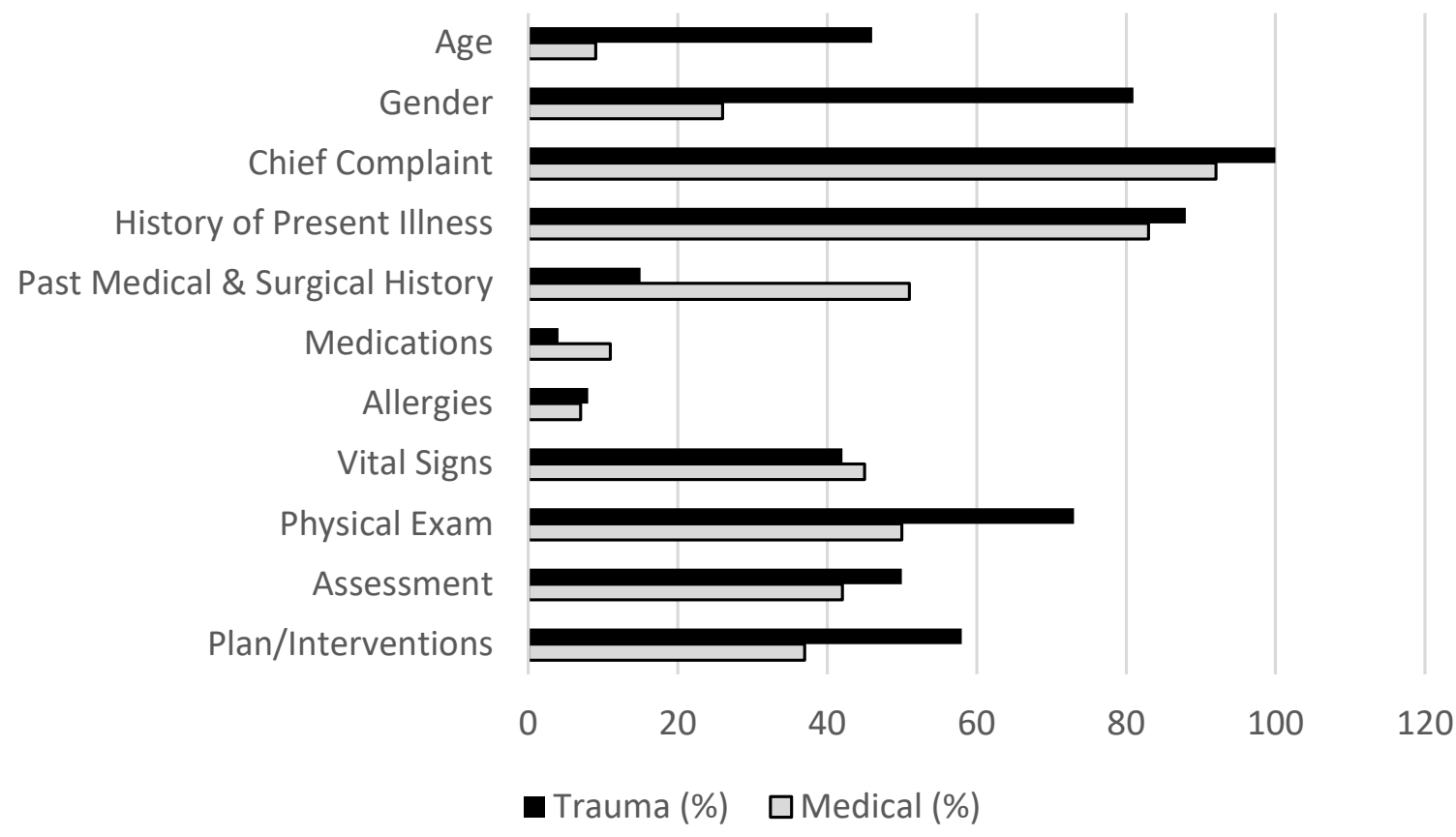

JCEMS · Volume $4 \cdot$ Issue $2 \cdot$ October 2021 
were observed concerning physical exam findings with a range of inclusion in handoffs $47-67 \%$ of the time. ${ }^{5,6}$

In contrast, the largest study $(\mathrm{n}=1091)$ available studying this same interaction showed that over $70 \%$ of the time EMS crews did report individual vital signs upon handoff. ${ }^{7}$ This represents a stark contrast with our study and the two mentioned previously. There are many possible explanations for this including but not limited to differences in state/local training of EMS and protocols within the specific ED. Another large contrast between our study and previous ones was the inclusion of age in handoffs. Whereas age was given in $19 \%$ of our handoffs it was given $41-84 \%$ of the time in other studies. ${ }^{5,6,7}$ When examining a subset of trauma patients, our findings are consistent with previous research, where age was reported $40 \%$ of the time. ${ }^{5}$ Interruptions and repeat questions occurred less often in our study when compared to another. ${ }^{6}$

Few studies have been conducted on the specific content of EMS handoffs. Those that have been performed previously have done so in individual locations such as ours. In general our data aligned with that of two other studies. As previous literature and review articles have pointed out there is a lack of data in this significant area of patient safety. ${ }^{9,10}$ Our data builds upon this already limited data while showing a general trend of incomplete patient handoffs. Objectively, we know that $80 \%$ of medical errors involve miscommunication during patient transfers and handoffs. ${ }^{8}$ Studies interviewing EMS personnel have shown that they have often felt the messages they are trying to relay to ED personnel are not heard, leading to perceived delays in care. ${ }^{11,12}$ Improvements in the relationship between EMS and ED personnel in the form of feedback have already shown significant improvement in the treatment of ischemic strokes. ${ }^{13}$ Documentation accompanying handoffs by way of a standardized checklist has been shown by a collegiate ambulance service to improve the completeness of handoffs. ${ }^{14}$ Understanding and improving this complex interaction between EMS and ED personnel is a modifiable patient safety concern for which better understanding is required.

Previous literature has highlighted the importance of structured tools and standardization of oral handoffs. ${ }^{9,10}$ Suggested improvements include better incorporation of electronic information boards with minimum requirements for patient handoffs in addition to interprofessional training so that providers from different backgrounds can better communicate and feel respected and listened to. ${ }^{10,12}$ Further studies could focus on implementing an interprofessional training program with local EMS providers and ED staff to ascertain if improvements can be made qualitatively. Studies could also question patient outcomes based on the quality of EMS handoffs.

\section{Limitations}

This study only took place between the hours of 0900-2000 Monday through Friday due to the observers' schedules. It is certainly possible that interactions could vary at different times of the day and late into the night, or during the weekends, leading specifically to selection bias.

During trauma bay resuscitations, it can be challenging to hear information, and some may have been missed by the observers.

Some information may have been transferred by a second EMS, by initial radio transmission, or by the patient, thereby being missed by the observer.

\section{Conclusion}

While patient reports from EMS to ED personnel frequently include chief complaint and history of the present illness other patient metrics are less commonly transferred. This represents possible weaknesses in the completeness of patient data received by ED personnel from EMS.

There appear to be opportunities to improve communication of certain key physical exam findings like vital signs.

Follow-up research could focus on ascertaining how the quality of EMS reports affects patient outcomes or on improving communication especially handoffs between EMS and the ED.

\section{Acknowledgements}

The authors report no conflicts of interest.

\section{References}

1. Rui P, Kang K, Ashman JJ. National Hospital Ambulatory Medical Care Survey: 2016 emergency department summary tables. 2016. Available from: https://www.cdc.gov/nchs/data/nhamcs/web_tables/2016_ed_web_tables. pdf

2. American College of Emergency Physicians, Emergency Nurses Association, National Association of EMS Physicians, National Association of Emergency Medical Technicians, National Association of State EMS officials. Transfer of patient care between EMS providers and receiving facilities. Prehosp Emer Care. 2014;18(2):503-503. doi:10.3109/10903127.2014.883001

3. United States. National Highway Traffic Safety Administration, and United States. Maternal Child Health Bureau. "First Responder: National Standard Curriculum (Instructor's Course Guide).” (1995). Web.

4. Lindsay LP, White ML, Sawyer K, Pruitt C, Wang H. Pilot survey of components of emergency medical services emergent handoffs. Ann Emerg Med. 2013;62(4). doi:10.1016/j.annemergmed.2013.07.111

5. Goldberg SA, Porat A, Strother CG, et al. Quantitative analysis of the content of EMS handoff of critically ill and injured patients to the Emergency Department. Prehosp Emerg Care. 2016;21(1):14-17. doi:10.1080/10903127 .2016 .1194930

6. Sumner BD, Grimsley EA, Cochrane NH, et al. Videographic assessment of the quality of EMS to Ed Handoff communication during pediatric resuscitations. Prehosp Emerg Care. 2018;23(1):15-21. doi:10.1080/1090312 7.2018.1481475

7. Panchal AR, Gaither JB, Svirsky I, Prosser B, Stolz U, Spaite DW. The impact of professionalism on transfer of care to the Emergency Department. J Emerg Med. 2015;49(1):18-25. doi:10.1016/j.jemermed.2014.12.062 
8. Joint Commission Center for Transforming Healthcare. Joint Commission Center for Transforming Healthcare Tackles Miscommunication Among Caregivers. Joint Commission Center for Transforming Healthcare, October $1,2017$.

9. Wood K, Crouch R, Rowland E, Pope C. Clinical handovers between Prehospital and Hospital Staff: Literature review. Emerg Med J. 2014;32(7):577581. doi:10.1136/emermed-2013-203165

10. Jensen SM, Lippert A, Østergaard D. Handover of patients: A topical review of ambulance crew to emergency department handover. Acta Anaesthesiol Scand. 2013;57(8):964-970. doi:10.1111/aas.12125

11. Meisel ZF, Shea JA, Peacock NJ, et al. Optimizing the patient handoff between Emergency Medical Services and the Emergency Department. Ann Emerg Med. 2015;65(3). doi:10.1016/j.annemergmed.2014.07.003

12. Bost N, Crilly J, Patterson E, Chaboyer W. Clinical handover of patients arriving by ambulance to a hospital emergency department: A qualitative study. Intern Emerg Nurs. 2012;20(3):133-141. doi:10.1016/j.ienj.2011.10.002

13. Choi B, Tsai D, McGillivray CG, Amedee C, Sarafin J-A, Silver B. Hospital-directed feedback to Emergency Medical Services improves prehospital performance. Stroke. 2014;45(7):2137-2140. doi:10.1161/ strokeaha.114.005679

14. Alatis A, Monahan B, Raymond A, Hudson K, Vieth J, Nable J. Checklists improve EMS Documentation: Quality Improvement in a collegiatebased EMS Agency. J Coll Emerg Med Serv. 2020;3(1):16-21. doi:10.30542/ jcems.2020.03.01.05

Author Affiliations: From University at Alabama at Birmingham - in Birmingham, AL (M.D.M, J.J.G., J.B., W.A.S.).

Address for Correspondence: Walter A. Schrading, MD, FACEP, FAWM | Email: wschrading@uabmc.edu
Conflicts of Interest/Funding Sources: By the JCEMS Submission Declaration Form, all authors are required to disclose all potential conflicts of interest and funding sources. All authors declared that they have no conflicts of interest. All authors declared that they did not receive funding to conduct the research and/or writing associated with this work.

Authorship Criteria: By the JCEMS Submission Declaration Form, all authors are required to attest to meeting the four ICMJE.org authorship criteria: (1) Substantial contributions to the conception or design of the work; or the acquisition, analysis, or interpretation of data for the work; AND (2) Drafting the work or revising it critically for important intellectual content; AND (3) Final approval of the version to be published; AND (4) Agreement to be accountable for all aspects of the work in ensuring that questions related to the accuracy or integrity of any part of the work are appropriately investigated and resolved.

Submission History: Received June 22, 2020; accepted for publication September $12,2021$.

Published Online: October 31, 2021

Published in Print: October 31, 2021 (Volume 4: Issue 2)

Reviewer Information: In accordance with JCEMS editorial policy, Original Research manuscripts undergo double-blind peer-review by at least two independent reviewers. JCEMS thanks the anonymous reviewers who contributed to the review of this work.

Copyright: @ 2021 Monaco, Grove, Beckedorf \& Schrading. This is an open access article distributed under the terms of the Creative Commons Attribution 4.0 International (CC BY 4.0) License, which permits unrestricted use, distribution, and reproduction in any medium, provided the original author and source are credited. The full license is available at: https://creativecommons.org/licenses/ by $/ 4.0 /$

Electronic Link: https://doi.org/10.30542/JCEMS.2021.04.02.03 